Z Gerontol Geriat 2019 · 52 (Suppl 4):S243-S248 https://doi.org/10.1007/s00391-019-01622-3 Eingegangen: 1. Juli 2019

Angenommen: 17. September 2019

๑ Der/die Autor(en) 2019

Die Anwendung freiheitsentziehender Maßnahmen ( $\mathrm{feM}$ ) bei Menschen mit kognitiven Beeinträchtigungen bzw. Demenz im Akutkrankenhaus ist noch immer allgemeine medizinische und pflegerische Praxis, aber ethisch alles andere als unumstritten. Zusätzlich zu klassischen medizinethischen Ansätzen zur Bewertung solcher Maßnahmen kann eine Analyse des Phänomens der Leiblichkeit neue und fruchtbare ethische Perspektiven offenlegen. Die Kategorie der Leiblichkeit kann helfen, Würde, Freiheit und Freiheitsentzug im klinischen Kontext neu zu denken und zu bewerten.

\section{Einleitung}

Die Anwendung von feM im Akutkrankenhaus ist von andauernder Aktualität $[15,19]$. Dabei sind diese Maßnahmen, zu denen beispielsweise Fixiergurte, Bettseitenteile und sedierende Medikamente zählen [15], nicht nur fachlich umstritten [1], sondern auch vor dem Hintergrund einer Ethik, die sich dem Schutz der menschlichen Würde und Freiheit

Sebastian Ritzi ist Doktorand und Kollegiat (ehem. Stipendiat) des interdisziplinären Graduiertenkollegs "Menschen mit Demenz im Akutkrankenhaus" am Netzwerk Alternsforschung der Universität Heidelberg, das von der Robert-Bosch-Stiftung gefördert wird. Die Autoren danken der Robert-Bosch-Stiftung für die Unterstützung des Promotionsprojektes. Bei diesem Beitrag handelt es sich um unabhängige Forschung. Die Ansichten, die in dieser Publikation wiedergegeben werden, sind die der Autoren.

\author{
S. Ritzi ${ }^{1,2} \cdot$ A. Kruse $^{1}$ \\ 'Institut für Gerontologie, Universität Heidelberg, Heidelberg, Deutschland \\ ${ }^{2}$ Institut für Gerontologie, Netzwerk Alternsforschung (NAR), Heidelberg, Deutschland
}

\title{
Würde, Freiheit, Leiblichkeit
}

\section{Ethische Kategorien bei der Anwendung freiheitsentziehender Maßnahmen bei Menschen mit Demenz im Akutkrankenhaus}

verschrieben hat, in den seltensten Fällen gerechtfertigt.

Sucht man nach ethischen Kriterien zur Bewertung von feMbei Menschen mit schweren kognitiven Beeinträchtigungen bzw. Demenz ${ }^{1}$, so lässt sich die klassische Prinzipienethik nach Beauchamp und Childress als "Minimalkonsens“ aller Heilberufe identifizieren [3, 25]. Moralische Probleme aus dem Spannungsfeld vierer Prinzipien - Autonomie, Schadensvermeidung, Patientenwohl und Gerechtigkeit - heraus zu bewerten, ist weithin etabliert. Was es im Anschluss an Beauchamp und Childress zu zeigen gilt, um ihren Ansatz weiter zu konkretisieren und auf die Problematik der feM anzuwenden, ist, dass sich dieses Spannungsfeld immer konkret am menschlichen Leib ereignet: Im Folgenden soll daher die Kategorie der Leiblichkeit, die in der Philosophie vergleichsweise jung ist, in der Debatte um feM bei Menschen mit Demenz im Akutkrankenhaus besonders hervorgehoben werden. Dabei soll diese nicht als gleichrangiges fünftes normatives Prinzip verstanden werden, sondern vielmehr als eine zentrale ethische Kategorie, die beschreibt, wie bzw. „wo" sich die menschliche Autonomie und Freiheit manifestieren. Betrachtet man die ethischen Konfliktfelder, die sich bei der Anwendung von feM eröffnen, im Lichte der Kategorie der Leiblichkeit, so zeigt

\footnotetext{
1 Aufgrund des Titels des von der RobertBosch-Stiftung geförderten Graduiertenkollegs "Menschen mit Demenz im Akutkrankenhaus" sowie aus Gründen der Lesbarkeit ist nachfolgend die Rede vom bzw. von „Menschen mit Demenz".
}

sich, dass diese Prinzipien nicht im luftleeren Raum schweben, sondern unmittelbar am Leib des Menschen ihren Ausdruck und ihre Anwendung finden.

Der nachfolgend skizzierte Ansatz ist von einem fach- und disziplinübergreifenden Paradigmenwechsel inspiriert, der in der Forschung als „,body turn“ [2], „corporeal turn“ [26] oder „embodied turn" [22] benannt wird. Der Begriff kann deskriptiv aufgefasst werden - im Sinne der Beschreibung, dass ein solcher bereits stattgefunden hat bzw. stattfindet -, hat aber auch eine normative und programmatische Komponente: $\mathrm{Er}$ soll stattfinden, d.h., Phänomene der Leiblichkeit sollen in den verschiedenen Wissenschaften neue Berücksichtigung finden.

\section{Die Kategorie der Leiblichkeit}

In der philosophischen Erschließung der Leiblichkeit ließen und lassen sich - von Husserl über Merleau-Ponty, Lévinas und Waldenfels bis hin zu heutigen Vertretern der Leibphänomenologie wie Thomas Fuchs - verschiedene formale und inhaltliche Schwerpunktsetzungen vornehmen. An dieser Stelle soll keine erschöpfende Rekonstruktion der Tradition der Leiblichkeitsphilosophie geleistet werden, vielmehr soll die spezifisch ethische Bedeutung des Leibes berührt und auf die Anwendung von feM im Akutkrankenhaus appliziert werden.

Anders als der Begriff „Körper“ (lat. corpus), der letztlich nichts anderes als ein dreidimensional ausgedehntes $\mathrm{Ob}$ jekt bezeichnet, steht „Leib“ (vom mit- 
telhochdeutschen lipp: Leben) für einen belebten bzw. beseelten Körper [4]. Wendet man diese Begriffe auf den Menschen an, so bezeichnet der Körper den sozusagen objektiven, physikalisch-biologischen Organismus des Menschen, während der Leib die subjektive und intersubjektive Art und Weise benennt, wie der Mensch in der Welt anwesend ist, wahrnimmt, erfährt, sich bewegt und sich verhält. Plessner brachte diesen Unterschied auf die berühmte Formel, der Mensch habe seinen Körper und sei sein Leib $[10,24]$. Eine besondere Schwierigkeit der philosophischen Erfassung des Phänomens Leib besteht darin, dass der Leib selbst eine Art Mittlerstellung einnimmt: Er ermöglicht dem Menschen Beziehungen zur Welt, bleibt dabei jedoch selbst sozusagen im Rücken. Das Auge ermöglicht z.B. das Sehen, kann dabei selbst aber nicht wirklich gesehen werden [11].

So wie der Leib in der Wahrnehmung als Wahrnehmungsmedium fungiert, so nimmt er in der menschlichen Handlung die Rolle des Handlungsmediums ein: Handeln, d.h. Verwirklichung und Manifestation der Freiheit nach außen, geschieht nur durch den Leib vermittelt: „Es ist diese Leiblichkeit, in deren Vollzug das Seelisch-Geistige erst wirklich wird: in Tat und Werk (anstatt bloß in der Möglichkeit des Gedankens und Mögens zu verbleiben), in der es erst Gestalt und Wirkmacht, Tatsächlichkeit erhält" [27, S. 248]. So kann die freie Entscheidung, einen bestimmten Ort zu erreichen, nur dadurch umgesetzt werden, dass sich das Subjekt vermittelt durch seinen Leib an denselben bewegt. Dasselbe gilt für intersubjektive Handlungen, die als solche auch immer zwischenleibliche sind: Will man einen anderen Menschen trösten, so kann dies immer nur vermittelt über den leiblichen Kontakt (Blick, nonverbale Kommunikation, Sprache, Berührung) realisiert werden. „[I]n der leiblichen Gestalt ... eines Menschen liegt selbst die Möglichkeit und in zunehmendem Maß die Verwirklichung, die Inkarnation seiner personalen Freiheit. Die Person realisiert sich nur durch ihren Leib ..." [9, S. 214]. Im berühmten Diktum Husserls heißt es entsprechend, der Leib ,ist Umschlagstelle von geistiger Kausalität in Naturkausalität“" [17, S. 286]: Die geistige
Kausalität, d.h. die Willensentscheidung, dieses oder jenes zu tun, verwirklicht sich durch den Leib nach außen in den Bereich der Natur. Die geistige Kausalität z. B., die Billardkugel zu ihrem Ziel zu bringen, wird durch den Leib in die Naturkausalität der Stoßbewegung des Armes übersetzt.

\section{Würde und Leiblichkeit}

Als Verwirklichungsort der Freiheit ist der Leib auch Träger und Versichtbarung der Würde des Menschen. So lässt sich aus der Menschenwürde eine eigene Würde des Leibes ableiten, die sich z.B. in dem Recht auf körperliche und seelische (man könnte auch zusammenfassend sagen leibliche) Unversehrtheit niederschlägt - ja, strenggenommen sind diese „zwei Würden“ gar nicht voneinander zu trennen. So erscheint es verkürzt zu sagen, man schade durch körperliche oder seelische Gewalt lediglich dem Leib einer Person und nicht der Person selbst. Folgt man der Analyse Fuchs' [9], so zeigt sich, dass Würde kein Abstraktum bleibt, sondern sich in der leiblichen Souveränität des Menschen äußert: Aufrechter Gang, erhobenes Haupt, gemessener Schritt usw. können sichtbar die an sich unsichtbare Souveränität der Person ausdrücken. Umgekehrt kann eine Würdeverletzung konkret Ausdruck in der leiblichen Erniedrigung und Brechung der Haltung des Menschen finden. Der Mensch büßt hier nichts an seiner intrinsischen und inhärenten Würde ein, die an sich unantastbar bleibt, jedoch kann sich diese nur noch gebrochen nach auBen manifestieren. Der Mensch hat hier die Verfügung(sgewalt) über seinen Leib teilweise oder gänzlich eingebüßt. Dies wiegt besonders schwer, da eine fundamentale Verwirklichung der Freiheit darin besteht, den eigenen Leib zu kultivieren und zu „beherrschen“. In der Fähigkeit etwa, das Gleichgewicht zu halten, die Ausscheidungen zu kontrollieren, die Triebe zu beherrschen und die Scham zu verbergen, drückt sich die Souveränität der Person aus; „, [d]ementsprechend gehört zu den massivsten Verletzungen der Würde nicht die Gewaltanwendung als solche, sondern die demütigende Brechung der leiblichen Souveränität" $[9$,
S. 210]. Neben allgemeinen Handlungsrichtlinien für die pflegerische und ärztliche Praxis - etwa die Zielsetzung, die leibliche Souveränität des Betroffenen zu wahren, schützen und fördern sowie die leibliche Kultivierung sicherzustellen ergeben sich aus dieser Betrachtung nun auch neue Überlegungen für die Anwendung von feM bei Menschen mit Demenz.

\section{Demenz, Leiblichkeit und freiheitsentziehende Maßnahmen}

Wie alle Maßnahmen, die unter das Konzept des wohltätigen Zwangs zu summieren sind, betreffen feM besonders Menschen mit Demenz, deren Verhalten oft als unvorhersehbar und herausfordernd bewertet wird [6]. Der Deutsche Ethikrat geht von der folgenden Definition von Zwang aus: ein Akteur überwindet den Willen einer adressierten Person. Dabei genüge schon die Überwindung einer Abwehr, in der sich der Wille des Adressaten manifestiere. Unter einem Willen wird hier die Fähigkeit einer Person verstanden, ,als Urheber ihre Handlungen selbstständig hervorzubringen und sie sich als eigene Handlungen zuzuschreiben" [6, S. 27]. Der wohltätige, also im Dienste des Menschen stehende Zwang erweist sich den Ausführungen des Deutschen Ethikrates zufolge „nicht als per se unzulässig, sondern kann unter bestimmten ... Bedingungen als Ultima Ratio bei der professionellen Sorge gerechtfertigt sein" [6, S. 10]. Der Deutsche Ethikrat differenziert dabei drei Fallkonstellationen [6, S. $10 \mathrm{f}$.]:

a) „Der Sorgeadressat äußert Wünsche und Bedürfnisse, ist aber in der konkreten Situation unzweifelhaft nicht zu einer freiverantwortlichen Entscheidung in der Lage. Für diese Personengruppe kann wohltätiger Zwang unter bestimmten Umständen gerechtfertigt sein."

b) „Der Sorgeadressat entscheidet, wobei in der vorliegenden Situation aber begründete Zweifel an der Freiverantwortlichkeit seiner Entscheidung bestehen." Schon in solchen Fällen könne wohltätiger Zwang u. U. gerechtfertigt sein. 
Z Gerontol Geriat 2019 · 52 (Suppl 4):S243-S248 https://doi.org/10.1007/s00391-019-01622-3

(c) Der/die Autor(en) 2019

\section{S. Ritzi · A. Kruse}

\section{Würde, Freiheit, Leiblichkeit. Ethische Kategorien bei der Anwendung freiheitsentziehender Maßnahmen bei Menschen mit Demenz im Akutkrankenhaus}

\section{Zusammenfassung}

Die Anwendung freiheitsentziehender Maßnahmen (feM) bei Menschen mit kognitiven Beeinträchtigungen bzw. Demenz im Akutkrankenhaus ist noch immer von Aktualität. Dabei sind diese Zwangsmaßnahmen fachlich nicht nur umstritten, sondern auch vor dem Hintergrund einer Ethik, die sich dem Schutz der Menschenwürde verschrieben hat, in den seltensten Fällen moralisch gerechtfertigt. Die ethische Bewertung von feM erfolgt zumeist in klassischen medizinethischen Paradigmen wie der Prinzipienethik nach Beauchamp und Childress. Um die Debatte an dieser Stelle anzureichern, wird nach einer kurzen Darstellung der Problematik die ethische
Kategorie der Leiblichkeit eingeführt. Dieses Phänomen, das seit einem "corporeal turn" in den Wissenschaften mehr und mehr Aufmerksamkeit erhält, kann neue Perspektiven auf Würde, Freiheit und Freiheitsentzug eröffnen: Freiheitsentziehende Maßnahmen finden nicht in einem luftleeren Raum der Ideen Anwendung, sondern am konkreten psychophysischen Leib des Menschen. Damit sind feM Eingriffe in die leibliche Souveränität des Menschen und richten sich gegen seine leiblich manifestierte Freiheit. Der Begriff des Leibes, der hier Anwendung findet, bezeichnet mehr als nur den physischen „Körper" und kann besonders gut die (inter)subjektive
Komponente des Phänomens beschreiben, die hier zu berücksichtigen ist. Zu prüfen ist abschließend auch, inwiefern der Freiheitsentzug durch medikamentöse Ruhigstellung einen besonders schwerwiegenden Eingriff in die menschliche Leiblichkeit darstellt. Ist die Kategorie der Leiblichkeit in die Debatte um feM eingeführt, können entscheidende neue Schwerpunktsetzungen vorgenommen werden.

Schlüsselwörter

Patientenversorgung · Persönliche Autonomie $\cdot$ Demütigung $\cdot$ Leibgedächtnis . Medizinische Ethik

\section{Dignity, freedom, embodiment. Ethical categories concerning the use of freedom-depriving measures in people with dementia in acute care settings}

\section{Abstract}

The use of freedom-depriving measures (physical and medicinal restraints) in people with cognitive impairment or dementia in clinical care settings is of ongoing importance. At the same time, these coercive measures are not only heavily debated but also in most cases ethically questionable from the perspective of the ethics of human dignity. Usually, the ethical evaluation of freedom-depriving measures follows classical paradigms of medical ethics, such as the Principles of Biomedical Ethics by Beauchamp and Childress. To enrich the debate at this point, the ethical category of embodiment ("Leiblichkeit") is introduced and discussed after a short summary of the ethical problem at hand. The phenomenon of the living body that has received increasingly more attention in several sciences since the proclaimed "corporeal turn" enables new perspectives towards human dignity, freedom and deprivation of freedom: freedom-depriving measures do not take place in an invisible realm of ideas but are directly applied to the psychophysical unity that is the living body of a person. Thus, freedom-depriving measures are an intervention into the bodily autonomy of the human being and the personal freedom that is manifested in the living body. The concept of the living body
("Leib") that is applied here, signifies more than just a physical object and is especially apt to capture the (inter)subjective dimension that has to be taken into account here. Finally, it will have to be investigated whether the use of medicinal restraints represents an especially serious interference into the sphere of human embodiment. Once introduced into the debate on freedom-depriving measures in clinical care, the category of embodiment can warrant decisive new emphases.

\section{Keywords}

Patient care · Personal autonomy · Humiliation . Body memory $\cdot$ Ethics, medical c) „Der Sorgeadressat entscheidet unzweifelhaft freiverantwortlich. In diesem Fall kann wohltätiger Zwang nicht gerechtfertigt sein - selbst in solchen Fällen nicht, wo dies aufseiten des Sorgeadressaten zu einer schweren Selbstschädigung und aufseiten der Sorgenden zu einer extremen Herausforderung ihrer professionellen Sorgeverbindlichkeiten führt".

Daraus erwachsen dem Deutschen Ethikrat zufolge zwei bedeutsame Konsequenzen: Zunächst sei eine Aussage darü- ber zu treffen, ob eine Person freiverantwortlich handelt oder nicht. Zudem, und dies ist für unsere Argumentation sehr wichtig, dürfe wohltätiger Zwang auch bei jenen Personen, die nicht freiverantwortlich handeln können, „nur unter bestimmten Voraussetzungen und nur als Ultima Ratio angewandt werden “ [6, S. 11].

Die Kategorie der Leiblichkeit in diese Problematik einzuführen, kann besonders deswegen fruchtbar sein, weil durch sie auch die Demenzerkrankung in einem neuen und weniger bedrohlichen Licht erscheint: Häufig wird der
Mensch mit einem rationalistischen und - um eine Formulierung Fuchs' aufzugreifen - „zerebrozentrischen“ [8] Menschenbild auf seinen Körper, genauer sein Gehirn und dessen Funktionsfähigkeit, reduziert. Ist diese dann durch eine Demenzerkrankung wesentlich beeinträchtigt, so scheint der Mensch diesem Menschenbild zufolge gerade das einzubüßen, das ihn zum Menschen macht: Die Demenz, schon ihrer ursprünglichen Etymologie nach eine "Abwesenheit“ von Geist (lat. mens), scheint den Menschen völlig zu dehumanisieren und entpersonalisieren [12, 20]. Auf dem Funda- 
ment eines solchen Reduktionismus' lieBe sich ein undifferenzierter und unreflektierter Umgang mit feM bei Menschen mit Demenz mit Leichtigkeit rechtfertigen: Zwangsmaßnahmen dieser Art beträfen schließlich statt eines leiblichen Würdesubjektes nur einen dysfunktionalen Organismus. „Doch diese Identifizierung unseres Selbstseins mit Kognition, Rationalität und Gedächtnis beruht letztlich auf einem dualistischen Konzept der Person ..., in dem der Körper nur als der Trägerapparat für den Geist bzw. für das Gehirn gilt. Der Kortex und das Denken werden damit zum Sitz der menschlichen Personalität, während der Rest des Körpers ebenso wie die verkörperten Gefühle... nur noch ein Schattendasein führen" [12, S. 49]. Der Dualismus, der hier kritisiert wird, ist seinem Ursprung nach kein anderer als der Descartes': Als zwei prinzipiell unterschiedene Substanzen klaffen für Descartes Körper (lat. res extensa) und Geist (lat. res cogitans) in einem unversöhnbaren Dualismus auseinander. Der Körper ist gänzlich getrennt vom Geist und letztlich nichts anderes als eine mechanistisch aufgefasste Gliedermaschine [5].

Der Blick auf die menschliche Leiblichkeit, die Körper und Geist umgreift, erlaubt einen holistischen Ansatz, der diese Verkürzungen vermeidet. Vor allem das Leibgedächtnis hilft in diesem $\mathrm{Zu}$ sammenhang zu verdeutlichen, dass die Identität und Kontinuität des Menschen nicht ausschließlich an dessen Gehirn und kognitive Fähigkeiten geknüpft sind. Das Leibgedächtnis bezeichnet die Art und Weise, wie die Biografie, die Erinnerungen, Erfahrungen und Gewohnheiten eines Menschen sich leiblich manifestieren, sei es in routinierten Bewegungsabläufen, im Zurechtfinden in vertrauter Umgebung, dem vertrauten Umgang mit Gegenständen, der Körpersprache, dem empathischen Ausdrucksverstehen, der Reaktion auf altvertraute Sinneswahrnehmungen etc. Es ist unumstritten, dass eines der deutlichsten Symptome der Alzheimer-Erkrankung der zunehmende Verlust des autobiografischen und semantischen Gedächtnisses ist. Umso bedeutender ist das Phänomen, dass weite Bereiche des Leibgedächtnis- ses noch bis in späte Stadien erhalten bleiben [12].

In diesem Zusammenhang ist zu betonen, dass im Leibgedächtnis Aspekte der Personalität, die in früheren Lebensaltern zentral für das Individuum waren, immer noch zu erkennen sind. Die Rede ist von „Inseln des Selbst“, einer Metapher, die besonders geeignet ist, die Kontinuitäten der Person zum Ausdruck zu bringen [20].

Mit Blick auf die oben getroffene Charakterisierung der Leiblichkeit als Manifestation der Freiheit kann das Leibgedächtnis so als ein Ausdruck dieser Inseln des Selbst beschrieben werden, in dem sich die freie Selbstbestimmung des Menschen mit Demenz in fragmentarischer Form äußert. Aus einer Betrachtung der Leiblichkeit des Menschen mit Demenz ergibt sich, dass sich nicht nur die Biografie desselben leiblich manifestiert vielmehr ist es die Freiheit des Menschen selbst, die im Leib in Erscheinung tritt. Der Mensch übt seine Freiheit in seinem Leib aus, was besonders in der Fortbewegungsfreiheit und in dem fundamentalen Recht auf körperlich-seelische (kurz: leibliche) Unversehrtheit zum Ausdruck kommt. Entsprechend schwer wiegt eine Beschränkung oder ein vollkommener Entzug dieser Freiheit, wie er in der Anwendung von feM vorliegen kann.

Vor diesem Hintergrund erscheinen feM als moralisch besonders problematisch, da sie eine Freiheit antasten, die den Menschen mit Demenz in seiner existenziellen Situation als psychophysische Einheit betrifft. Es bedarf dementsprechend einer deutlichen Reflexion darüber, dass feM einen Eingriff in - man könnte fast sagen - die letzte Freiheit darstellen können, die dem Betroffenen noch bleibt.

Mit Blick auf die Intention hinter der Anwendung von feM sei hier nicht unterstellt, diese seien primär als Einschränkung der leiblich manifesten Freiheit bzw. leiblichen Souveränität der Betroffenen intendiert. Zunächst einmal sind strukturelle Gründe anzuführen, die wesentlich dazu beitragen können, dass Menschen mit Demenz im Akutkrankenhaus von feM betroffen sind: Nicht nur mangelnde Kenntnisse über eine adäquate, ganzheitliche medizinisch-pflegerische Versorgung von
Menschen mit Demenz, sondern auch eine erschwerende Zeit-, Personal- und Ressourcenknappheit sind im klinischen Alltag mitentscheidend, ob auf Zwangsmaßnahmen wie feM zurückgegriffen wird [23, 29]. Dies nicht zuletzt aus dem Grund, dass in der Organisation Akutkrankenhaus noch häufig ökonomische Interessen leitend sind oder zumindest in Konkurrenz mit dem pflegerischmedizinischen Versorgungsauftrag der Institution treten [23].

Neben strukturellen Gründen ist es in vielen Fällen der vermeintliche Schutz vor körperlichen Schäden z.B. infolge von Sturzereignissen, der die beteiligten Akteure im Akutkrankenhaus dazu verleitet, feM einzuleiten. Davon abgesehen, dass fachwissenschaftlicher Konsens darüber herrscht, dass feM zur Vermeidung von Sturzereignissen eher ungeeignet und somit kontraindiziert sind $[7,13,28]$, kann eine solche paternalistische Haltung letztlich in einer eigenen Art der Demütigung resultieren. Im Anschluss an Margalit, der in seiner Politik der Würde verschiedene Strukturen der institutionellen Demütigung identifiziert [21], lässt sich argumentieren, dass Menschen mit Demenz häufig aus einem falsch verstandenen Fürsorge- oder sogar Barmherzigkeitsmotiv übermäßig beschützt, reglementiert und mithin ihrer Freiheit beraubt werden was ebenso eine Demütigung darstellt wie z.B. die Vorenthaltung bestimmter Dienst- und (medizinischer) Versorgungsleistungen [20]. Im Kontext der Anwendung von feM sei an dieser Stelle v. a. auf die Nicht-Bereitstellung alternativer Handlungsmittel (z. B. Niedrigflurbett) hingewiesen. Man läuft Gefahr, dass die Anwendung von feM zugleich ein demütigendes „Zuviel“ an paternalistischer Bevormundung und ein demütigendes "Zuwenig“ an adäquater medizinischpflegerischer Versorgung darstellt.

In dem Maße, in dem eine feM in die leiblich manifeste Freiheit des Menschen mit Demenz eingreift, spitzt sich auch die Gefahr der Brechung der leiblichen Souveränität zu. Wurde bisher von feM meistens implizit als mechanischen Maßnahmen gesprochen, soll nun die Aufmerksamkeit auf Maßnahmen gerichtet werden, die auf den ersten Blick weitaus 
weniger eingreifend erscheinen - die Gabe von Medikamenten mit dem Primärziel der Ruhigstellung [18]. Solche sind an dieser Stelle ausdrücklich nicht zu verwechseln mit einer therapeutisch indizierten Medikation, bei der eine Ruhigstellung als Nebenwirkung in Kauf genommen wird. Neben mechanischen begleiten chemische Formen der Freiheitsentziehung die medizinisch-pflegerische Versorgung von Menschen mit Demenz im Akutkrankenhaus [15, 18]. Sie sind schon lange nicht mehr die Ausnahme und oft gestützt von der fachlich und moralisch fragwürdigen Vorstellung, personelle Mängel zu kompensieren - obgleich sie mit Stürzen und einem erhöhten Betreuungsbedarf assoziiert sind [16].

In der ethischen Bewertung des Freiheitsentzugs durch den Einsatz von Psychopharmaka bei Menschen mit Demenz, die zurzeit noch als Desiderat detaillierter künftiger Forschung zu bewerten ist, kann die Einführung der Kategorie der Leiblichkeit neue Aspekte der Problematik erhellen. So können Ansätze, die noch in den traditionellen Paradigmen operieren [14], weiter vertieft und ergänzt werden. Zwar wirkt der Effekt einer medikamentösen $\mathrm{Ru}$ higstellung von außen friedlich und sanft - v.a. im Vergleich zu mechanischen Fixierungen -, jedoch liegt hier letztlich ein schwerwiegenderer Eingriff in die menschliche Leiblichkeit vor, insofern diese Maßnahme nicht äußerlich bleibt, sondern in das Innere des Menschen eindringt. Ruft man sich die Husserl'sche Definition des Leibes als Umschlagstelle von geistiger Kausalität in Naturkausalität in Erinnerung, so wird deutlich, dass eine medikamentöse Intervention, die das Bewusstsein des Menschen reduziert, die Möglichkeit dieser Vermittlungsfunktion des Leibes aufhebt. Das menschliche Bewusstsein verliert durch die medikamentöse $\mathrm{Ru}$ higstellung zumindest teilweise seine freie Verfügung(sgewalt) über den Leib. Die Freiheit der betroffenen Person, die sich besonders in der Leiblichkeit und hier noch einmal in hoch individueller Art und Weise im Leibgedächtnis zu erkennen geben möchte, wird an ihrer Verwirklichung gehindert.

\section{Fazit für die Praxis}

- Die Anwendung von freiheitsentziehenden Maßnahmen (feM) bedarf einer ethischen Prüfung, die neben klassischen prinzipienethischen Ansätzen auch die ethische Kategorie der Leiblichkeit berücksichtigen sollte.

- Dualistische und zerebrozentrische Menschenbilder sollten mithilfe einer Reflexion auf die psychophysische Einheit des menschlichen Leibes zugunsten einer holistischen Sicht des Menschen revidiert werden.

- Freiheitsentziehende Maßnahmen greifen in die leiblich manifeste Freiheit des Menschen mit Demenz ein und stellen eine Form der Demütigung und Brechung der leiblichen Souveränität dar. Alternative Handlungskonzepte sind stets auszuschöpfen.

- Die Gabe von Medikamenten mit dem Primärziel der Ruhigstellung stellt aus ethischer Perspektive eine äußerst schwerwiegende Form des Freiheitsentzugs und der Demütigung dar.

\section{Korrespondenzadresse}

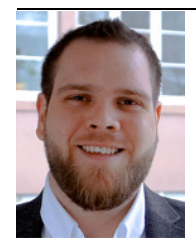

S. Ritzi

Institut für Gerontologie, Netzwerk Alternsforschung (NAR) Bergheimer Straße 20, 69115 Heidelberg, Deutschland sebastian.ritzi@ gero.uni-heidelberg.de

\section{Einhaltung ethischer Richtlinien}

Interessenkonflikt. S. Ritzi und A. Kruse geben an, dass kein Interessenkonflikt besteht.

Für diesen Beitrag wurden von den Autoren keine Studien an Menschen oder Tieren durchgeführt. Für die aufgeführten Studien gelten die jeweils dort angegebenen ethischen Richtlinien.

The supplement containing this article is not sponsored by industry.

Open Access. Dieser Artikel wird unter der Creative Commons Namensnennung 4.0 International Lizenz (http://creativecommons.org/licenses/by/4.0/deed. de) veröffentlicht, welche die Nutzung, Vervielfäl- tigung, Bearbeitung, Verbreitung und Wiedergabe in jeglichem Medium und Format erlaubt, sofern Sie den/die ursprünglichen Autor(en) und die Quelle ordnungsgemäß nennen, einen Linkzur Creative Commons Lizenz beifügen und angeben, ob Änderungen vorgenommen wurden.

\section{Literatur}

1. Abraham J, Ralph M, Köpke S (2019) Freiheitsentziehende Maßnahmen in der Pflege. Public Health Forum 27(1):75-77

2. Alloa E, Bedorf T, Grüny C et al (2012) Leiblichkeit: Geschichte und Aktualität eines Konzepts. UTB, Stuttgart

3. Beauchamp TL, Childress JF (2013) Principles of Biomedical Ethics. OUP, Oxford

4. Borsche T, Kaulbach F (1980) Leib, Körper. In: Gründer JRK (Hrsg) Historisches Wörterbuch der Philosophie. Schwabe, Basel, Stuttgart, S173-185

5. Descartes R (2008) Meditationes de prima philosophia /Meditationen über die Grundlagen der Philosophie: Lateinisch-deutsch. Felix Meiner, Hamburg

6. Deutscher Ethikrat (2018) Hilfe durch Zwang? Professionelle Sorgebeziehungen im Spannungsfeld von Wohl und Selbstbestimmung. Stellungnahme Deutscher Ethikrat, Berlin

7. Evans D, Wood J, Lambert L (2003) Patient injury and physical restraint devices: a systematic review. JAdv Nurs 41:274-282

8. Fuchs T (2010) Das Leibgedächtnis in der Demenz. In: Kruse A (Hrsg) Lebensqualität bei Demenz? Zumgesellschaftlichen undindividuellen Umgang mit einer Grenzsituation im Alter. Akademische Verlagsgesellschaft AKA, Heidelberg, S 231-242

9. Fuchs T (2008) Die Würde des menschlichen Leibes. In: Härle W, Vogel B (Hrsg) Begründung von Menschenwürde und Menschenrechten. Herder, Freiburg, Basel, Wien, S202-219

10. Fuchs T (2015) Körper haben oder Leib sein. Gesprächspsychotherapie Pers Berat 3:147-153

11. Fuchs T (2018) Leib, Raum, Person: Entwurf einer phänomenologischen Anthropologie. Klett-Cotta, Stuttgart

12. Fuchs $T$ (2018) Leiblichkeit und personale Identität in der Demenz. DZPhil 66(1):48-61

13. Hamers JP, Huizing AR (2005) Why do we use physical restraints in the elderly? Z Gerontol Geriat 38:19-25

14. Heide R (2018) Psychopharmaka als Mittel zur Freiheitsbeschränkung: Ethische Bewertung für Medizin, Pflege und Pharmazie. Springer, Wiesbaden

15. Hendlmeier I, Bickel H, Hessler JB et al (2018) Demenzsensible Versorgungsangebote im Allgemeinkrankenhaus. Z Gerontol Geriat 51:509-516

16. Hewer W, Thomas C (2017) Psychopharmakotherapie bei Demenz und Delir. Im Spannungsfeld zwischen Leitlinienempfehlungen und Versorgungspraxis.ZGerontol Geriat 50:106-114

17. Husserl E (1952) Ideen zu einer reinen Phänomenologie und phänomenologischen Philosophie. Zweites Buch: Phänomenologische Untersuchungen zur Konstitution. Nijhoff, Den Haag

18. Isfort M, Klostermann J, Gehlen D et al (2014) Pflegethermometer 2014. Eine bundesweite Befragung von leitenden Pflegekräften zur Pflege und Patientenversorgung von Menschen mit Demenz im Krankenhaus. Deutsches Institut für angewandte Pflegeforschung e. V., Köln

19. Krüger C, Mayer H, Haastert B et al (2013) Use of physical restraints in acute hospitals in Germany: 


\section{Fachnachrichten}

a multi-centre cross-sectional study. Int J Nurs Stud 50:1599-1606

20. Kruse A (2017) Lebensphase hohes Alter: Verletzlichkeit und Reife. Springer, Berlin, Heidelberg

21. Margalit A (2012) Politik der Würde: über Achtung und Verachtung. Suhrkamp, Berlin

22. Nevile M (2015) The embodied turn in research on language and social interaction. Res Lang Soc Interact 48:121-151

23. Newerla A (2017) Demenz als kritisches Moment: Ordnungsversuche im Akutkrankenhaus. ZME 63:193-204

24. Plessner H (1970) Lachen und Weinen: eine Untersuchung nach den Grenzen menschlichen Verhaltens. In: Plessner H (Hrsg) Philosophische Anthropologie. Fischer, Frankfurt, S11-171

25. Riedel A, Behrens J, Giese C et al (2017) Zentrale Aspekte der Ethikkompetenz in der Pflege. Ethik Med 29:161-165

26. Sheets-Johnstone M (2015) The corporeal turn: an interdisciplinary reader. Imprint Academic, Exeter

27. Splett J (2002) Leibhaftige Freiheit. ZME 48:247-257

28. Sze TW, Leng CY, Lin SK (2012) The effectiveness of physical restraints in reducing falls among adults in acute care hospitals and nursing homes: a systematic review. JBI Libr Syst Rev 10:307-351

29. Wunder M (2017) Selbstbestimmung und Demenz - Herausforderung für die Behandlung dementer Patienten im Krankenhaus. ZME 63:205-215

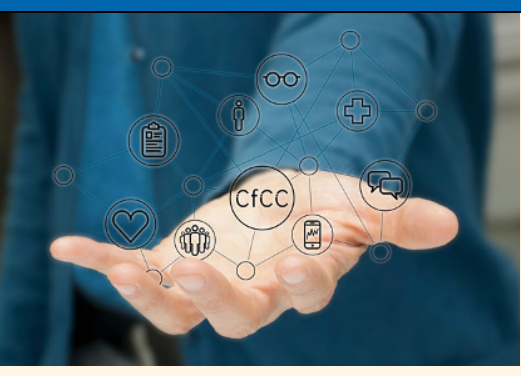

Geförderter Auslandsaufenthalt durch die Robert Bosch Stiftung

Care for Chronic Condition (CfCC)

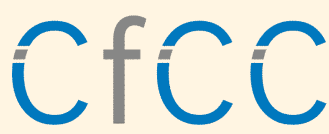

Care for Chronic Condition

Mit dem internationalen Hospitations- und Fortbildungsprogramm "Care for Chronic Condition" (CFCC) fördert die Robert Bosch Stiftung Bildungsaufenthalte im Ausland. Dafür bewerben können sich Fach- und Führungskräfte aus sämtlichen Gesundheitsberufen. Voraussetzung ist: Sie versorgen Menschen mit chronischen und mehrfachen Erkrankungen. Teilnehmende erhalten Zuschüsse zu Hospitations- und Fortbildungsgebühren sowie zu Reise- und Aufenthaltskosten. Außerdem unterstützt CfCC den Transfer von im Ausland gewonnenen Erkenntnissen in den Arbeitsalltag.

Interessierte können über zwei Wege am CfCC-Programm teilnehmen: Sie können zum einen eine Förderung für einen selbst recherchierten Bildungsaufenthalt beantragen. Oder sie bewerben sich auf konkrete vom CfCC-Team vermittelte Bildungsaufenthalte. Aktuelle Angebote finden sich unter www.cfcc-programm.de/angebote. Voraussetzung für eine Bewerbung ist eine aussagekräftige Fragestellung und eine Transferidee sowie eine Befürwortung des Arbeitgebers. Es sind sowohl Team- als auch Einzelbewerbungen möglich. Ausführliche Informationen zum Bewerbungsverfahren und den Link zum OnlineBewerberportal finden Interessierte unter www.cfcc-programm.de/bewerbung. Das CfCC-Team steht jederzeit für Fragen per Telefon oder E-Mail zur Verfügung www.cfcc-programm.de/kontakt. 